\title{
Use of Butyl-Methyl Methacrylate Thin-Sections for Quantification of mRNA Using Branched-DNA ISH
}

\author{
Andrew J. Bowling ${ }^{1}$ and Heather E. Pence ${ }^{1}$ \\ ${ }^{1 .}$ Dow AgroSciences, Indianapolis, IN.
}

New high-sensitivity in situ hybridization (ISH) methods, such as branched DNA (bDNA) ISH, allow the detection of single mRNA molecules [1]. One of the benefits conferred by single-molecule sensitivity is the ability to quantify the number of molecules of a particular mRNA by simple counting. This works very well for transcripts that exist at low to moderate levels in the cells. However, because the individual foci formed by the enzymatic deposition of chromogen or fluorophores fill a volume larger than the actual mRNA occupies, if the mRNA density is sufficiently high, the margins of the chromogen spots will merge, making the discrimination of single mRNA particles difficult or impossible.

One possible way to overcome this is through the use of thinner sections. ISH is normally applied to formaldehyde fixed, paraffin embedded (FFPE) sections, which are on the order of 5-12 $\mu \mathrm{m}$ in thickness. It is technically very difficult to cut paraffin sections thinner than about 2-3 microns. Plastic sections, however, can easily be cut much thinner than this. Removal of the supporting medium from tissue sections improves ISH results by allowing the oligo probes access to the mRNA in the tissue. Unfortunately, most plastic embedding resins are not removable, which is one of the main benefits of paraffin sections. One resin that is both easy to cut into thin sections and is removable with a brief acetone treatment is butylmethyl methacrylate (BMM). This resin formulation was originally developed to allow immunocytochemical localization of cytoskeletal proteins in plant sections [2]. We have found that this resin is amenable to the production of sub-micron sections that yield good mRNA signals with the branched DNA ISH method, RNAscope. To show the effect of section thickness on quantification of abundant transcripts, the highly-expressed gene phosphoenolpyruvate carboxylase (PEPC) in corn leaf was used. This gene has previously been shown to be heavily expressed in corn leaf [3]. Another use of serial thin sections is analysis of 3D organization of the tissue through the use of serial section 3D reconstruction [4], and we expect to demonstrate the ability of this resin to produce array-tomographylike ISH data in the near future.

Corn plants were grown in a greenhouse under standard conditions. Small rectangles $(2 \times 5 \mathrm{~mm})$ of leaf blade were embedded in butyl-methyl methacrylate following the method of Baskin et al. [2]. Briefly, leaf pieces were fixed overnight in 4\% formaldehyde in PBS, dehydrated in a graded ethanol series, infiltrated with a graded series 3:1 butyl:methyl methacrylate (Polysciences), changed to neat 3:1 BMM with 0.1\% benzoin methyl ether (BME) as the catalyst, and polymerized by exposure to UV light for 16 hours in gelatin capsules. All steps were done at $4^{\circ} \mathrm{C}$. Blocks were trimmed and faced with a DiATOME diamond trimming blade, and thin sections and ribbons were cut with a DiATOME HistoJumbo diamond knife. Sections were mounted on Fisher SuperFrost Plus slides and de-plasticized with a simple 10 min acetone treatment. Slides were probed for PEPC mRNA using RNAscope VS from ACD on a Ventana Discovery Ultra automated slide staining system [3].

PEPC mRNAs were detected in BMM thin sections of corn leaf, demonstrating that this resin is fully compatible with branched DNA ISH methods (Figure 1). The improved preservation of the tissue by the BMM resin over paraffin allowed better visualization of the sub-cellular localization of individual PEPC mRNA molecules. By using a series of sections at different sub-micron thicknesses, the abundant PEPC 
signal was resolved into single mRNA particles. Imaging of single mRNA particles on these thin sections allowed the direct quantification of PEPC signal in mesophyll cells of the corn leaf. BMM thin-sections can be used to resolve single mRNA molecules from the large aggregates commonly seen in paraffin sections when probing for highly-abundant genes. Furthermore, this resin should be amenable to the reconstruction of 3D images from serial sections. Taken together, this would yield high resolution 3D localization data of mRNAs.

\section{References:}

[1] F Wang et al, Journal of Molecular Diagnostics 14 (2012), p. 22.

[2] T. Baskin et al, Planta 187, p. 405.

[3] A. Bowling et al, Appl Plant Sci. 2 (2014), p. 1.

[3] K. D. Micheva and S. J. Smith, Neuron 55 (2007), p. 25.
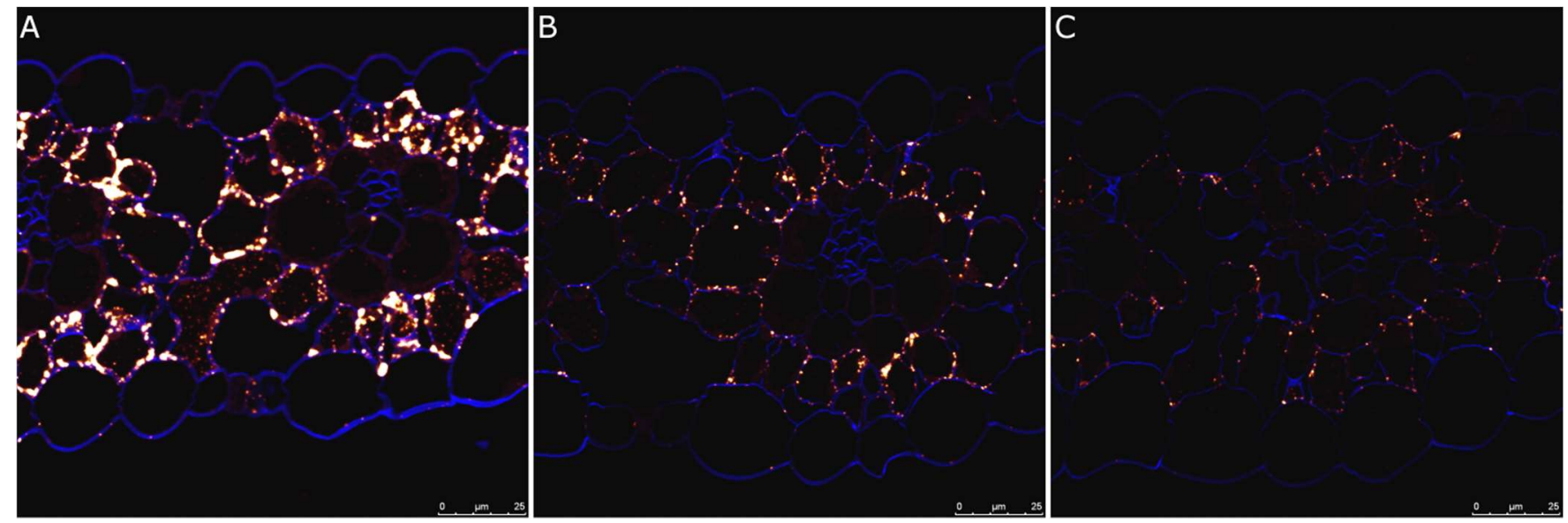

Figure 1. Corn leaf sections, cut to different thicknesses, probed for PEPC mRNA. A.) A $1 \mu$ m-thick section showing a very high level of mRNA signal, which is not resolvable into single mRNA molecules. B.) A $0.5 \mu \mathrm{m}$ BMM thin section showing the excellent level of structural detail visible in BMM sections. At this thickness, most of the PEPC mRNAs are resolvable as single spots. C.) At $0.25 \mu \mathrm{m}$ thickness, nearly all of the PEPC mRNAs in the tissue section are resolved into single spots. 